\title{
Diagonal series of rational functions (several variables)
}

\author{
by SŁawomir Cynk and Piotr Tworzewski (Kraków)
}

\begin{abstract}
We give representations of Nash functions in a neighbourhood of a polydisc (torus) in $\mathbb{C}^{m}$ as diagonal series of rational functions in a neighbourhood of a polydisc (torus) in $\mathbb{C}^{m+1}$.
\end{abstract}

1. Introduction. Let $\Omega$ be an open subset of $\mathbb{C}^{m}$. We shall use the following notation:

$\mathcal{O}(\Omega)$ - the space of all holomorphic functions on $\Omega$,

$\mathcal{N}(\Omega)$ - the space of all Nash functions on $\Omega$,

$\mathcal{R}(\Omega)$ - the space of all rational holomorphic functions on $\Omega$.

For any compact subset $K$ of $\mathbb{C}^{m}$ we denote by $\mathcal{O}(K)$ the space of all functions defined on $K$ which have a holomorphic extension to an open neighbourhood of $K$. In the same way we define $\mathcal{N}(K)$ and $\mathcal{R}(K)$. We denote by $U$ and $T$ the unit disc and unit circle in $\mathbb{C}$ respectively.

We consider the diagonal operator

$$
\mathcal{D}: \mathcal{O}\left(T^{m} \times T\right) \ni f \rightarrow \mathcal{D}(f) \in \mathcal{O}\left(T^{m}\right),
$$

defined by

$$
\mathcal{D}(f)(z)=\sum_{\alpha \in \mathbb{Z}^{m}} a_{\alpha,|\alpha|} z^{\alpha}
$$

where

$$
f(z, w)=\sum_{\alpha \in \mathbb{Z}^{m}, n \in \mathbb{Z}} a_{\alpha, n} z^{\alpha} w^{n}
$$

is the Laurent expansion of $f$ (see [2]-[5], [7], [9]).

1991 Mathematics Subject Classification: Primary 32A05, 32A25.

Key words and phrases: diagonal series, rational function, Nash function, rationally convex, Hadamard convolution.

Supported by KBN Grant 210779101. 
In Section 3 we shall prove our main results (Theorems (3.1) and (3.2)):

$$
\mathcal{D}\left(\mathcal{R}\left(T^{m} \times T\right)\right)=\mathcal{N}\left(T^{m}\right), \quad \mathcal{D}\left(\mathcal{R}\left(\bar{U}^{m} \times \bar{U}\right)\right)=\mathcal{N}\left(\bar{U}^{m}\right) .
$$

The case $m=1$ of these theorems can be found in [3]. A local version of Theorem (3.2) was proved in [5] by completely algebraic methods.

Section 2 is of preparatory nature. The most important in this section is Lemma (2.5) which plays a crucial role in the proofs of our main results.

In Section 4 we study relations between diagonals and Hadamard convolution of power series of one variable. In particular, in Example (4.3), we construct an algebraic Laurent series with transcendental regular part.

2. Preliminary results. Let $\Omega$ be an open subset of $\mathbb{C}^{m}$ and let $g \in$ $\mathcal{O}(\Omega)$.

(2.1) Definition. We say that $g$ is a Nash function at $x_{0} \in \Omega$ if there exist an open neighbourhood $V$ of $x_{0}$ in $\Omega$ and a polynomial $P: \mathbb{C}^{m} \times \mathbb{C} \rightarrow \mathbb{C}$, $P \neq 0$, such that $P(x, g(x))=0$ for $x \in V$. The function $g$ is said to be a Nash function on $\Omega$ if it is a Nash function at each point of $\Omega$. We denote by $\mathcal{N}(\Omega)$ the space of all Nash functions on $\Omega$.

The basic information about Nash functions may be found in [3], [10]. Let us only state the following

(2.2) Remark. Let $\Omega$ be an open connected subset of $\mathbb{C}^{m}$ and let $f \in \mathcal{N}(\Omega)$. Then the closure $\bar{f}^{\mathrm{Z}}$ of $f$ in the Zariski topology of $\mathbb{C}^{m} \times \mathbb{C}$ is an irreducible algebraic hypersurface in $\mathbb{C}^{m} \times \mathbb{C}$.

Let $K$ be a compact subset of $\mathbb{C}^{m}$.

(2.3) Definition. We say that $K$ is rationally convex if for each $z_{0} \notin K$ the following two equivalent conditions hold:

(1) There exists a rational function $R \in \mathcal{R}\left(K \cup\left\{z_{0}\right\}\right)$ such that $\left|R\left(z_{0}\right)\right|>$ $\|R\|_{K}$.

(2) There exists a polynomial $P: \mathbb{C}^{m} \rightarrow \mathbb{C}$ such that $P\left(z_{0}\right)=0$ and $P(z) \neq 0$ for every $z \in K$.

(2.4) Re mark. From [2, Remark 1.1] it follows that for any compact sets $A_{1}, \ldots, A_{n}$ in $\mathbb{C}$ the product $A_{1} \times \ldots \times A_{n}$ is rationally convex. For more information about rationally convex sets we refer the readers to [2], [6].

We end this section with the main lemma of [3], which gives a special representation of Nash functions.

(2.5) Lemma (S. Cynk, cf. [3]). Let $E$ be a compact, rationally convex set and let $D$ and $G$ be connected open subsets of $\mathbb{C}^{m}$ such that $\emptyset \neq G \subset E \subset D$. 
Let a be a fixed point of $G$. If $g \in \mathcal{N}(D)$ then there exists a function $h \in \mathcal{N}(G)$ and polynomials $P, Q: \mathbb{C}^{m} \times \mathbb{C} \rightarrow \mathbb{C}$ such that
(1) $h(a)=0$
(2) $h(G) \subset U$,
(3) $\bar{h}^{\mathrm{Z}} \cap(G \times \bar{U})=h$,
(4) $Q^{-1}(0) \cap(G \times \bar{U})=\emptyset$,
(5) $g(z)=P(z, h(z)) / Q(z, h(z))$ for $z \in G$.

3. Diagonal operator. In this section we consider the diagonal operator

$$
\mathcal{D}: \mathcal{O}\left(T^{m} \times T\right) \rightarrow \mathcal{O}\left(T^{m}\right)
$$

defined by (1.1). The operator $\mathcal{D}$ admits the integral representation

$$
\mathcal{D}(f)(z)=\frac{1}{2 \pi i} \int_{T} f\left(\frac{z}{w}, w\right) \frac{d w}{w}, \quad z \in T^{m} .
$$

(3.1) Theorem. $\mathcal{D}\left(\mathcal{R}\left(T^{m} \times T\right)\right)=\mathcal{N}\left(T^{m}\right)$.

Proof. Take $f \in \mathcal{R}\left(T^{m} \times T\right)$ and define $f_{1}(z, w):=f(z / w, w)$ for $(z, w) \in T^{m} \times T$. Then $f_{1} \in \mathcal{R}\left(T^{m} \times T\right)$. Therefore there exist polynomials $P, Q: \mathbb{C}^{m} \times \mathbb{C} \rightarrow \mathbb{C}$ such that for each $(z, w) \in T^{m} \times T$ we have $Q(z, w) \neq 0$ and $f_{1}(z, w)=P(z, w) / Q(z, w)$. There exists an open connected neighbourhood $D$ of the set $T^{m}$ such that $Q^{-1}(0) \cap(D \times T)=\emptyset$. Put $\widetilde{f}_{1}(z, w)=P(z, w) / Q(z, w)$ for $(z, w) \in D \times T$.

There exist a non-empty open subset $D_{1}$ of $D$ and a system of Nash functions $\Phi_{1}, \ldots, \Phi_{k} \in \mathcal{N}\left(D_{1}\right)$ with pairwise disjoint graphs such that $\{(z, w) \in$ $\left.D_{1} \times U: Q(z, w) w=0\right\}=\Phi_{1} \cup \ldots \cup \Phi_{k}$ (where $U$ is the unit disc in $\mathbb{C}$ ).

Using the above equation and the residue formula we get

$$
\begin{array}{r}
\frac{1}{2 \pi i} \int_{T} \tilde{f}_{1}(z, w) \frac{d w}{w}=\sum_{j=1}^{k} \frac{1}{N !} \frac{\partial^{N}}{\partial w^{N}}\left(\left(w-\Phi_{j}(z)\right)^{N+1} \frac{P(z, w)}{Q(z, w) w}\right)\left(z, \Phi_{j}(z)\right), \\
z \in D_{1},
\end{array}
$$

where $N$ is a sufficiently large integer. Consequently, the basic properties of Nash functions imply that

$$
D_{1} \ni z \mapsto \frac{1}{2 \pi i} \int_{T} f\left(\frac{z}{w}, w\right) \frac{d w}{w} \in \mathbb{C}
$$

is a Nash function. Hence $\mathcal{D}(f) \in \mathcal{N}\left(T^{m}\right)$.

In order to prove the opposite inclusion fix $g \in \mathcal{N}\left(T^{m}\right)$. There exist $\delta \in\left(0, \frac{1}{3}\right)$ and $\widetilde{g} \in \mathcal{N}\left(A^{m}(1-3 \delta, 1+3 \delta)\right)$ such that $\widetilde{g} \mid T^{m}=g$ (where $A^{m}(r, R):=\left\{z \in \mathbb{C}^{m}: r<\left|z_{i}\right|<R, i=1, \ldots, m\right\}$, for $\left.0 \leq r \leq R\right)$. 
By Lemma (2.5) and Remark (2.4) there exist a Nash function $h \in$ $\mathcal{N}\left(A^{m}(1-2 \delta, 1+2 \delta)\right)$ and polynomials $P, Q: \mathbb{C}^{m} \times \mathbb{C} \rightarrow \mathbb{C}$ such that

(1) $h\left(A^{m}(1-2 \delta, 1+2 \delta)\right) \subset U$,

(2) $\bar{h}^{\mathrm{Z}} \cap\left(A^{m}(1-2 \delta, 1+2 \delta) \times \bar{U}\right)=h$,

(3) $Q^{-1}(0) \cap\left(A^{m}(1-2 \delta, 1+2 \delta) \times \bar{U}\right)=\emptyset$,

(4) $\widetilde{g}(z)=P(z, h(z)) / Q(z, h(z))$ for $z \in A^{m}(1-2 \delta, 1+2 \delta)$.

Let $R$ be an irreducible polynomial describing the graph of $h$. There exists $\varepsilon>0$ such that $R^{-1}(0) \cap\left(A^{m}(1-\delta, 1+\delta) \times \Delta(1+\varepsilon)\right)=h \mid A^{m}(1-\delta, 1+\delta)$ and $Q^{-1}(0) \cap\left(A^{m}(1-\delta, 1+\delta) \times \Delta(1+\varepsilon)\right)=\emptyset$ (where $\Delta(r):=\{z \in \mathbb{C}:$ $|z|<r\}$, for $r>0)$.

Defining

$$
f(z, w):=w \cdot \frac{P(w z, w)}{Q(w z, w)} \cdot \frac{R_{w}(w z, w)}{R(w z, w)}
$$

we get $f \in \mathcal{R}\left(T^{m} \times T\right)$ and

$\mathcal{D}(f)(z)=\frac{1}{2 \pi i} \int_{T} \frac{P(z, w)}{Q(z, w)} \cdot \frac{R_{w}(z, w)}{R(z, w)} d w=\frac{P(z, h(z))}{Q(z, h(z))}=g(z), \quad z \in T^{m}$.

Thus $\mathcal{D}(f)=g$ and the proof is complete.

In view of the inclusions $\mathcal{O}\left(\bar{U}^{m} \times \bar{U}\right) \subset \mathcal{O}\left(\bar{T}^{m} \times \bar{T}\right)$ and $\mathcal{O}\left(\bar{U}^{m}\right) \subset \mathcal{O}\left(\bar{T}^{m}\right)$ we can consider the operator

$$
\mathcal{D}: \mathcal{O}\left(\bar{U}^{m} \times \bar{U}\right) \rightarrow \mathcal{O}\left(\bar{U}^{m}\right) .
$$

We end this section with

(3.2) Theorem. $\mathcal{D}\left(\mathcal{R}\left(\bar{U}^{m} \times \bar{U}\right)\right)=\mathcal{N}\left(\bar{U}^{m}\right)$.

Pr o of. Since $\mathcal{D}\left(\mathcal{R}\left(\bar{U}^{m} \times \bar{U}\right)\right) \subset \mathcal{N}\left(\bar{U}^{m}\right)$ is a direct consequence of Theorem (3.1) it is sufficient to prove the reverse inclusion.

Fix $g \in \mathcal{N}\left(\bar{U}^{m}\right)$. There exist $\delta \in(0,1)$ and $\widetilde{g} \in \mathcal{N}\left(\Delta^{m}(1+4 \delta)\right)$ such that $\widetilde{g} \mid \bar{U}^{m}=g$.

By Lemma (2.5) there exist $h \in \mathcal{N}\left(\Delta^{m}(1+3 \delta)\right)$ and polynomials $P, Q$ : $\mathbb{C}^{m} \times \mathbb{C} \rightarrow \mathbb{C}$ such that

(1) $h(0)=0$,

(2) $h\left(\Delta^{m}(1+3 \delta)\right) \subset U$,

(3) $\bar{h}^{\mathrm{Z}} \cap\left(\Delta^{m}(1+3 \delta) \times \bar{U}\right)=h$,

(4) $Q^{-1}(0) \cap\left(\Delta^{m}(1+3 \delta) \times \bar{U}\right)=\emptyset$,

(5) $\widetilde{g}(z)=P(z, h(z)) / Q(z, h(z))$ for $z \in \Delta^{m}(1+3 \delta)$.

Let $R$ be an irreducible polynomial describing the graph of $h$. There exists $\varepsilon>0$ such that $R^{-1}(0) \cap\left(\Delta^{m}(1+2 \delta) \times \Delta(1+\varepsilon)\right)=h \mid \Delta^{m}(1+2 \delta)$, $Q^{-1}(0) \cap\left(\Delta^{m}(1+2 \delta) \times \Delta(1+\varepsilon)\right)=\emptyset$, and $(1+\varepsilon)(1+\delta)<1+2 \delta$. 
Defining

$$
f(z, w):=w \cdot \frac{P(w z, w)}{Q(w z, w)} \cdot \frac{R_{w}(w z, w)}{R(w z, w)}
$$

we get $f \mid \bar{U}^{m} \times T \in \mathcal{R}\left(\bar{U}^{m} \times T\right)$ and $\mathcal{D}(f)=g$.

From the above construction we see that $R(z, w)=(w-h(z)) A(z, w)$, where $A$ is a holomorphic function on $\Delta^{m}(1+2 \delta) \times \Delta(1+\varepsilon)$ which does not vanish at any point. Hence

$$
f(z, w)=\frac{P(w z, w)}{Q(w z, w)} \cdot \frac{R_{w}(w z, w)}{A(w z, w)} \cdot \frac{1}{1-h(w z) / w}
$$

for $(z, w) \in \Delta^{m}(1+\delta) \times \Delta(1+\varepsilon)$.

But $h(0)=0$ so $h(w z) / w \in \Delta^{m}(1+\delta) \times \Delta(1+\varepsilon)$. Therefore, by the Schwarz Lemma,

$$
\left|\frac{h(w z)}{w}\right| \leq \frac{1}{1+\varepsilon} \quad \text { for }(z, w) \in \Delta^{m}(1+\delta) \times \Delta(1+\varepsilon)
$$

and consequently $f \in \mathcal{R}\left(\bar{U}^{m} \times \bar{U}\right)$, and the proof is complete.

4. Hadamard convolution. Let us recall that the Hadamard convolution of power series $f(z)=\sum_{n=0}^{\infty} a_{n} z^{n}$ and $g(z)=\sum_{n=0}^{\infty} b_{n} z^{n}$ is defined to be $(f * g)(z)=\sum_{n=0}^{\infty} a_{n} b_{n} z^{n}$ (cf. [1]). It is closely connected with our diagonal operator in the case $m=1$.

Since for $f, g \in \mathcal{R}(\bar{U})$ we have $(f * g)(z)=\mathcal{D}(f(z) g(w))$, it follows from Theorem (3.2) that $f * g \in \mathcal{N}(\bar{U})$. Applying the residue formula one can prove the following stronger result ([1, Ths. 5.1.2 and 5.2.2]).

(4.1) Theorem. If $f, g \in \mathcal{R}(\bar{U})$ then $f * g \in \mathcal{R}(\bar{U})$. If $f \in \mathcal{R}(\bar{U})$, $g \in \mathcal{N}(\bar{U})$ then $f * g \in \mathcal{N}(\bar{U})$.

Using the formula $(f * g)(z)=\mathcal{D}(f(z) g(w))$ we can define the Hadamard convolution of functions $f, g \in \mathcal{O}(T)$. Then $(f * g)(z)=\sum_{n \in \mathbb{Z}} a_{n} b_{n} z^{n}$, where $f(z)=\sum_{n \in \mathbb{Z}} a_{n} z^{n}$ and $g(z)=\sum_{n \in \mathbb{Z}} b_{n} z^{n}$.

(4.2) Remark. Under the above definition it is easy to check that the first part of Theorem (4.1) remains true. The second statement is false in this case. This will be shown by Example (4.3) and the following observation.

If $f(z)=\sum_{n \in \mathbb{Z}} a_{n} z^{n}$ and $g(z)=(1-z / 2)^{-1}$ then $(f * g)(z)=$ $\sum_{n \in \mathbb{N}} a_{n}(z / 2)^{n}=f_{1}(z / 2)$ where $f_{1}(z)=\sum_{n \in \mathbb{N}} a_{n} z^{n}$ is the regular part of the series $\sum_{n \in \mathbb{Z}} a_{n} z^{n}$.

(4.3) ExAmple. Let

$$
f(z):=\left[\left(1-\frac{z}{2}\right)\left(1-\frac{1}{2 z}\right)\right]^{-1 / 2}
$$


be the holomorphic branch of the square root of the function $[(1-z / 2) \times$ $(1-1 /(2 z))]^{-1}$ in the annulus $A(1 / 2,2)$ such that $f(1)=2$. Then $f \mid T \in$ $\mathcal{N}(T)$. We shall show that the regular part $f_{1}$ of $f$ is transcendental.

Observe that $f$ satisfies the differential equation $\left(2 z^{3}-5 z^{2}+2 z\right) f^{\prime}(z)=$ $\left(1-z^{2}\right) f(z)$. Hence, comparing coefficients in Taylor expansions we see that $f_{1}$ satisfies

$$
\left(2 z^{3}-5 z^{2}+2 z\right) f_{1}^{\prime}(z)=\left(1-z^{2}\right) f_{1}(z)+a z+b,
$$

for some complex numbers $a$ and $b$.

Consider the function $C(z):=f_{1}(z) / f(z)$. From (4.4) we get $C^{\prime}(z)=$ $R(z) f(z)$ where $R(z)=-(a z+b) /\left(4 z^{2}\right)$ is a rational function.

Now, suppose, on the contrary, that $f_{1}$ is a Nash function. Then also $C$ is a Nash function and therefore $C$ is an algebraic element over the field $\mathbb{C}(z)$ of rational functions. Let $F:=\mathbb{C}(z)(f)$ be the field generated by $f$ over $\mathbb{C}(z)$. Since $f$ is algebraic over $\mathbb{C}(z)$ of degree 2 , the set $\{1, f\}$ is a basis of the field $F$ over $\mathbb{C}(z)$. The derivative $f^{\prime}$ belongs to $F$ so $F$ is closed under differentiation.

Since $C$ is algebraic over $\mathbb{C}(z)$, it is also algebraic over $F$. Let $P(C)=$ $C^{n}+a_{1}(z) C^{n-1}+\ldots+a_{n}(z)=0$ be the minimal polynomial of $C$ over $F$. Differentiating the above equation we get

$$
\left(a_{1}^{\prime}+n C^{\prime}\right) C^{n-1}+\ldots+\left(a_{n-1} C^{\prime}+a_{n}^{\prime}\right)=0 .
$$

Since $C^{\prime} \in F$ it follows that $n C^{\prime}+a_{1}^{\prime}=0$ and hence $C \in F$. Consequently, $f_{1}=f C \in F$. So there exist rational functions $R_{1}, R_{2} \in \mathbb{C}(z)$ such that $f_{1}(z)=R_{1}(z)+R_{2}(z) f$.

As $f_{1}$ is holomorphic in $\Delta(2)$ and $f$ is not meromorphic in this disc we get $R_{2}=0$. Thus $f_{1}(z)=R_{1}(z)$ and consequently $f(z)=f_{1}(z)+f_{1}(1 / z)-$ $f_{1}(0)=R_{1}(z)+R_{1}(1 / z)-a_{0}$, so $f$ is a rational function. This contradicts the definition of $F$, and so $f_{1}$ is transcendental.

\section{References}

[1] L. Bieberbach, Analytische Fortsetzung, Springer, Berlin, 1967.

[2] S. Cynk, Representations of Nash functions, Astérisque, to appear.

[3] S. Cynk and P. Tworzewski, Diagonal series of rational functions, Ann. Polon. Math. 55 (1991), 57-63.

[4] P. Deligne, Intégration sur un cycle évanescent, Invent. Math. 76 (1984), 129-143.

[5] J. Denef and L. Lipshitz, Algebraic power series and diagonals, J. Number Theory 26 (1987), 46-67.

[6] B. A. Fuks, Special Topics in the Theory of Analytic Functions of Several Complex Variables, Fizmatgiz, Moscow, 1963 (in Russian).

[7] H. Furstenberg, Algebraic functions over finite fields, J. Algebra 7 (1967), 271277 . 
[8] S. Łojasiewicz, Introduction to Complex Analytic Geometry, Birkhäuser, Basel, 1991.

[9] K. V.Safonov, On conditions for algebraicity and rationality of the sum of a power series, Mat. Zametki 41 (3) (1987), 325-332 (in Russian).

[10] P. Tworzewski, Intersections of analytic sets with linear subspaces, Ann. Scuola Norm. Sup. Pisa (4) 17 (2) (1990), 227-271.

INSTITUTE OF MATHEMATICS

JAGIELLONIAN UNIVERSITY

REYMONTA 4

30-059 KRAKÓW, POLAND

Reçu par la Rédaction le 28.4.1993 